\title{
The Vanishing Pituitary Tumor: A Case Report
}

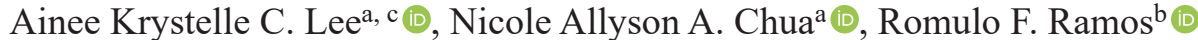

\begin{abstract}
Prolactinomas are the most common functional pituitary tumors and present with elevated serum prolactin levels. This may or may not be accompanied by mass-related clinical symptoms. Dopamine agonists are its principal treatment. Data on prolactinoma remission and relapse after treatment withdrawal are limited. Here we report a patient presenting with headache, amenorrhea, galactorrhea, visual field impairments and a high serum prolactin level. After a definitive diagnosis of pituitary macroadenoma, the patient was treated with bromocriptine. Twelve months after treatment, tumor size markedly reduced, there was resolution of symptoms and patient was eventually lost to follow-up. After 10 months without treatment, tumor recurred. Bromocriptine was resumed for 5 more years and discontinued thereafter. Since then, the patient has been asymptomatic for the past 12 years; surveillance imaging showed no tumor recurrence with annual prolactin level all within normal range. This case adds to the limited data confirming that dopamine agonists in patients with prolactinomas can be successfully discontinued with a high remission rate, provided that there is adequate duration of treatment and sufficient follow-up.
\end{abstract}

Keywords: Pituitary tumor; Pituitary macroadenoma; Prolactinoma; Bromocriptine; Dopamine agonist

\section{Introduction}

Prolactinomas are the most common functional adenomas and occur most frequently among women aged 20 - 50 years [1]. These tumors are derived from lactotroph cells in the pituitary gland secreting prolactin [2]. The more common effects manifested by prolactinomas include headache and visual field impairment, loss of libido, infertility, osteoporosis in both sex-

Manuscript submitted June 3, 2021, accepted July 5, 2021

Published online July 25, 2021

anternal Medicine Residency Program, Cardinal Santos Medical Center, San Juan, Philippines

bSection of Endocrinology, Department of Internal Medicine, Cardinal Santos Medical Center, San Juan, Philippines

${ }^{\mathrm{c} C}$ Corresponding Author: Ainee Krystelle C. Lee, Internal Medicine Residency Program, Cardinal Santos Medical Center, San Juan, Philippines.

Email: aineekrystellelee@gmail.com

doi: https://doi.org/10.14740/jem753 es, menstrual irregularities and galactorrhea in females, and erectile dysfunction in males $[1,3]$. Dopamine agonists are the principal treatment, but data on prolactinoma remission and relapse after treatment withdrawal remain limited $[1,4]$.

\section{Case Report}

\section{Investigations}

This case is of a 60-year-old, G5P5, married female who consulted due to a 1-year history of amenorrhea shortly after delivering her fifth child. She was 35 years old at the time of symptom presentation. This was accompanied by headache, blurring of vision and copious milky nipple discharge despite breastfeeding for only 3 months.

\section{Diagnosis}

Diagnostics done were as follows: 1) serum prolactin assay elevated at $500 \mathrm{ng} / \mathrm{mL}$; 2) left temporal hemianopsia with right superior quadrantanopia on visual field perimetry; and 3) cranial magnetic resonance imaging (MRI) showing a 2.0 $\mathrm{cm}$ suprasellar mass without compression of the optic chiasm nor invasion of the cavernous sinus. Official copies of the MRI film and visual field perimetry are not included in this report as data storage policies have lapsed making the films irretrievable. Further hematological testing revealed normal serum cortisol, adrenocorticotropic hormone (ACTH), thyroidstimulating hormone (TSH), T4, growth hormone $(\mathrm{GH})$, and insulin-like growth factor-1(IGF-1), with a low serum luteinizing hormone (LH) and normal follicle-stimulating hormone (FSH).

\section{Treatment}

The patient was initially started on bromocriptine $2.5 \mathrm{mg}$, half tablet thrice daily. Bromocriptine was started over cabergoline as the latter was not available in the country.

\section{Follow-up and outcomes}

Patient had weekly follow-up, and immediately after the first week of treatment, there was marked improvement in visual 


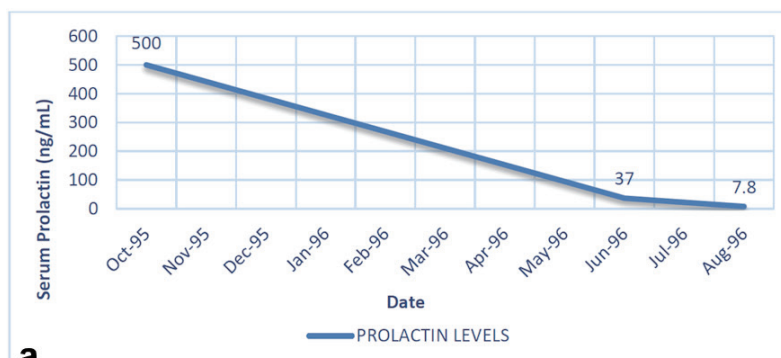

a

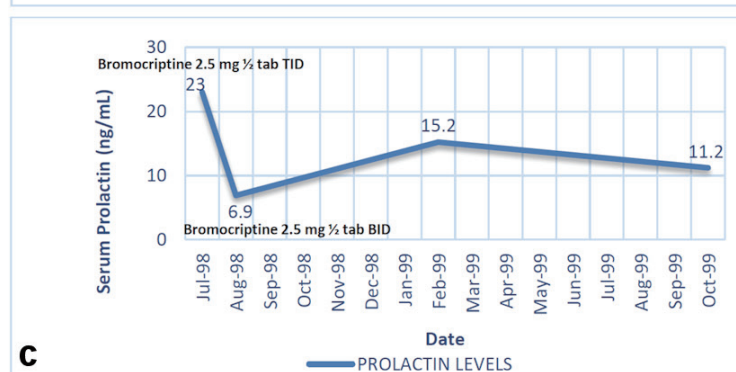

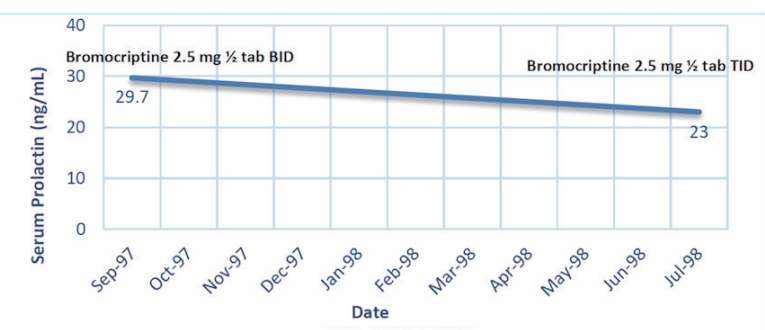

b

PROLACTIN LEVELS

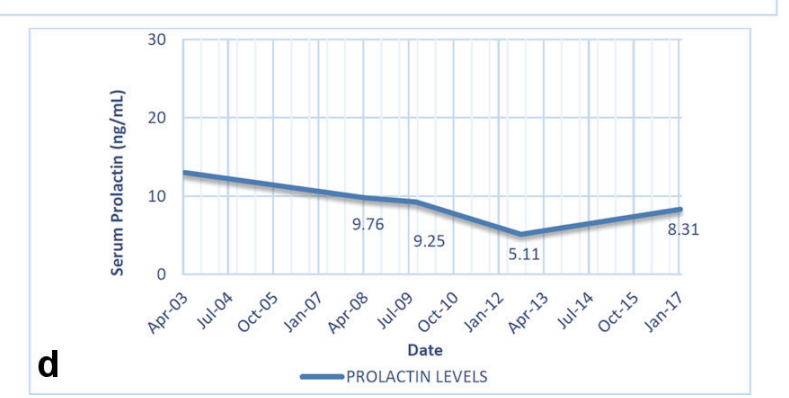

Figure 1. (a) Outcome of prolactin levels with bromocriptine use from initial consultation to 10 months of treatment (October 1995 to August 1996). (b) Outcome of prolactin levels with bromocriptine resumption from September 1997 to July 1998,10 months into second treatment. (c) Monitoring of prolactin levels with corresponding dose adjustment of bromocriptine. (d) Monitoring of prolactin levels after discontinuation of bromocriptine. Serum prolactin normal range: $0.8-16.0 \mathrm{ng} / \mathrm{mL}$.

field perimetry. There was resolution of the left temporal hemianopsia and right superior quadrantanopia with visual acuity now at 20/70. On her 3rd month of bromocriptine treatment still dosed at $2.5 \mathrm{mg}$, half tablet thrice daily, there was resumption of her regular menses.

Eight months into treatment, serum prolactin levels notably decreased to $37 \mathrm{ng} / \mathrm{mL}$ as seen in Figure 1a. On the 10th month of treatment, serum prolactin levels decreased to 7.8 $\mathrm{ng} / \mathrm{mL}$, which is within the normal range of 0.8 to $16.0 \mathrm{ng} /$ $\mathrm{mL}$.

A repeat cranial MRI after 12 months of treatment revealed no tumor evidence, hence concluding that the patient had resolution of prolactinoma. The patient then discontinued bromocriptine and was lost to follow-up. Ten months after discontinuation of medication, patient had recurrence of symptoms again presenting with amenorrhea. Workup done showed tumor recurrence on computed tomography (CT) cranial imaging with dimension of $0.4 \times 0.6 \mathrm{~cm}$, and an elevated serum prolactin level at $29.7 \mathrm{ng} / \mathrm{mL}$. Once again, the patient was treated with bromocriptine at $2.5 \mathrm{mg}$ tablet, half tablet twice daily. Serial monitoring of prolactin levels was done and on follow-up after 11 months of treatment, prolactin level still elevated at $23.0 \mathrm{ng} / \mathrm{mL}$; hence, bromocriptine dose was increased to $2.5 \mathrm{mg}$ tablet, half tablet thrice daily as shown in Figure 1b.

Serum prolactin level measurements were monitored regularly. On the 11th month of the second treatment cycle, prolactin normalized to $6.9 \mathrm{ng} / \mathrm{mL}$, hence bromocriptine dose was decreased to twice daily from thrice daily as seen in Figure 1c.

After 5 years of treatment, a repeat cranial MRI showed a normal-sized pituitary gland with no tumor evidence. Bromocriptine was then discontinued. The patient is currently asymptomatic for the past 12 years; surveillance imaging re- vealed no tumor recurrence and serial serum prolactin level measurements since then were all normal as seen in Figure 1d.

\section{Discussion}

In the treatment and management of prolactinomas, pharmacological therapy with dopamine agonist remains to be the gold standard. However, surgical removal and radiotherapy can prove to be equally effective options especially in cases where prolactinomas are resistant to treatment $[1,5]$. In the case presented above, intervention done was pharmacologic therapy.

Among the dopamine agonists, cabergoline is preferred over bromocriptine due to its higher efficacy rate with a lower incidence of adverse effects $[1,6]$. Furthermore, results of various studies show that bromocriptine decreases pituitary tumor size by $50 \%$ in two-thirds of patients, whereas cabergoline exhibits a larger $90 \%$ tumor size reduction in the same population. This case did not adhere to the standard treatment due to unavailability of the standard drug.

The patient was lost to follow-up and discontinued treatment after 12 months. Higher remission rates may be correlated to longer treatment duration before trying dopamine agonist withdrawal, but studies remain to be inconsistent. Prolactinoma recurrence is most commonly seen during the first 6 to 12 months following cessation of treatment, and recurrence rates can be as high as $93 \%$ for macroprolactinomas and $64 \%$ for microprolactinomas [3]. Dopamine agonists can be safely discontinued in cases of prolactinomas with a tumor volume reduction of more than $50 \%$ without tumor recurrence [7]. Various studies have different methodologies regarding the exact duration of therapy prior to treatment withdrawal, but all are consistent that longer duration of therapy leads to 
lesser tumor recurrence $[3,8]$. Patients with microadenomas and those with macroadenomas and a negative MRI scan after treatment are good candidates for drug withdrawal. Because tumor enlargement is uncommon in small tumors, it is not necessary to obtain a prewithdrawal MRI in a patient with microadenoma [9]. The best candidates for dopamine agonist withdrawal are patients who have no visible tumor on MRI, have a nadir prolactin level $<1.0 \mathrm{ng} / \mathrm{dL}$ during drug treatment, and those who received drug treatment for more than 6 years [8]. Among patients successfully treated with dopamine agonists, follow-up after 24 months of treatment withdrawal showed that approximately $60-70 \%$ remained in remission $[4,8]$. As of this writing, there are no definite guidelines with regards to the duration of surveillance of prolactin levels among patients in remission. Prospective studies are needed to define the optimal surveillance duration after treatment withdrawal in both macro- and microprolactinomas as recurrence rates are different for both $[9,10]$.

\section{Conclusions}

Long-term and adequate administration of dopamine agonists for the treatment of prolactinomas leads to regression of the tumor size up to its complete disappearance. This case adds to the limited data confirming that dopamine agonists in patients with prolactinomas can be discontinued with a high remission rate, provided that there is adequate duration of treatment and sufficient follow-up.

\section{Acknowledgments}

The authors would like to acknowledge the patient for allowing this case to be published.

\section{Financial Disclosure}

None to declare.

\section{Conflict of Interest}

There is no conflict of interest in the publication of this manuscript.

\section{Informed Consent}

The patient was given informed consent for the publishing of her case information.

\section{Author Contributions}

Ainee Krystelle C. Lee: principal investigator, case writer and editor, data curation, formal analysis, and validation. Nicole Allyson A. Chua: second lead investigator, case writer, data curation. Romulo F. Ramos: project conceptualization, data curation, and methodology

\section{Data Availability}

The authors declare that data supporting the findings of this study are available within the article.

\section{References}

1. Molitch ME. Diagnosis and treatment of pituitary adenomas: a review. JAMA. 2017;317(5):516-524.

2. Rahmanian M, Meybodi HA, Larijani B, Mohajeri-Tehrani MR. Giant prolactinoma: case report and review of literature. J Diabetes Metab Disord. 2013;12(1):3.

3. Wong A, Eloy JA, Couldwell WT, Liu JK. Update on prolactinomas. Part 1: Clinical manifestations and diagnostic challenges. J Clin Neurosci. 2015;22(10):1562-1567.

4. Teixeira M, Souteiro P, Carvalho D. Prolactinoma management: predictors of remission and recurrence after dopamine agonists withdrawal. Pituitary. 2017;20(4):464470.

5. Wong A, Eloy JA, Couldwell WT, Liu JK. Update on prolactinomas. Part 2: Treatment and management strategies. J Clin Neurosci. 2015;22(10):1568-1574.

6. Melmed S, Casanueva FF, Hoffman AR, Kleinberg DL, Montori VM, Schlechte JA, Wass JA, et al. Diagnosis and treatment of hyperprolactinemia: an Endocrine Society clinical practice guideline. J Clin Endocrinol Metab. 2011;96(2):273-288.

7. Xia MY, Lou XH, Lin SJ, Wu ZB. Optimal timing of dopamine agonist withdrawal in patients with hyperprolactinemia: a systematic review and meta-analysis. Endocrine. 2018;59(1):50-61.

8. Ji MJ, Kim JH, Lee JH, Lee JH, Kim YH, Paek SH, Shin $\mathrm{CS}$, et al. Best candidates for dopamine agonist withdrawal in patients with prolactinomas. Pituitary. 2017;20(5):578584.

9. Schlechte JA. Long-term management of prolactinomas. J Clin Endocrinol Metab. 2007;92(8):2861-2865.

10. Barber TM, Kenkre J, Garnett C, Scott RV, Byrne JV, Wass JA. Recurrence of hyperprolactinaemia following discontinuation of dopamine agonist therapy in patients with prolactinoma occurs commonly especially in macroprolactinoma. Clin Endocrinol (Oxf). 2011;75(6):819-824. 